Th 


\section{Schriftsteller in der Massengesellschaft}

Zur politischen Essayistik und Publizistik Heinrich und Thomas Manns, Kurt Tucholskys und Ernst Jüngers während der $Z$ wanziger Jahre 
Die in [ ] erscheinenden Zahlen verweisen auf die Anmerkungen

Die vorliegende Arbeit wurde im Jahre 1972 von der Philosophischen Fakultät der Universität des Saarlandes als Dissertation angenommen. Professor Helmut Kreuzer sei für seine hilfreiche Förderung der Arbeit gedankt. Die zu ihrer Entstehung notwendige materielle Basis schuf ein Promotionsstipendium des Evangelischen Studienwerkes Villigst.

ISBN 978-3-476-00273-0

ISBN 978-3-476-03023-8 (eBook)

DOI 10.1007/978-3-476-03023-8

(C) Springer-Verlag GmbH Deutschland 1973

Ursprünglich erschienen bei J. B. Metzlersche Verlagsbuchhandlung und Carl Ernst Poeschel Verlag GmbH in Stuttgart 1973. 


\section{Inhalt}

Einleitung . . . . . . . . . . . . . . . . . . . . . .

Ästhetische Fixierung der deutschen Essayforschung - Ludwig Rohners normativ orientierte Gattungsbestimmung - Konsequenzen ästhetisch normativ ausgerichteter Forschung am Beispiel von Urteilen über Heinrich Manns Essayistik - Publikumsbezug des Essays - Methodische Vorbemerkungen zum betrachteten Zeitraum der Weimarer Republik und zur Auswahl der im Mittelpunkt stehenden Autoren - Aufbau der Kapitel des Hauptteils

\section{Aligemeiner Teil}

I. Stichworte zur gesellschaftlichen Entwicklung vom Kaiserreich zur Weimarer Republik: Massengesellschaft - Massendemokratie . . . . . .

Wahlrecht und Wahlbeteiligung vor und nach dem Ersten Weltkrieg - Wahl des Staatsoberhauptes nach der Weimarer Verfassung - Hintergrund der Massendemokratie: Entwicklung vom Agrarstaat zur industriellen Massengesellschaft

II. Die negative Stellung der literarischen Intelligenz zur gesellschaftlichen Entwicklung bis zum Ende des Ersten Weltkriegs . . . . . . . . . 6

1. Jahrhundertwende . . . . . . . . . . . . . . . . . 6

Aisthetizismus und Individualismus der Stilkunst um 1900 - Heimatkunst als Opposition zur gesellschaftlichen Entwicklung

2. Aktivismus

Expressionismus und Aktivismus - Kurt Hillers Programm - Rubiners realitätsferne Position - Scheitern der Aktivisten nach dem November 1918 - Verhältnis der Aktivisten zur Masse - Hillers Unterscheidung von "Demos « und "Ochlos« und sein Konzept eines "Herrenhauses« - Hermann Brochs pessimistische Anerkennung der Massendemokratie - Tollers Antithese "Masse - Mensch «

III. Die unterschiedliche Revision des negativen Massenbegriffs in marxistischem und nichtmarxistischem Denken der zwanziger Jahre . . . . .

1. Marxistische Schriftsteller . . . . . . . . . . . . . . . . 12

a) Dialektik von Masse und Individuum . . . . . . . . . . . . 12

Proletkultische Kritik am Aktivismus - Johannes R. Bechers Forderung an die Intellektuellen - Brechts "Mann ist Mann - Brechts Massenbegriff im "Badener Lehrstück vom Einverständnis « 
b) Literarische Konsequenzen . . . . . . . . . . . . . . . .

Darstellung der Masse im proletkultischen Schauspiel in Rußland - Deutsche Massenschauspiele - Arbeiterkulturwoche Leipzig 1924 - Sprechchorwerk Rolle des Chors in Brechts "Flug der Lindberghs « und der "Maßnahme* Zusammenfassende Bemerkung

2. Nichtmarxistische Autoren . . . . . . . . . . .

a) Masse als Summe nivellierter Individuen . . . . . . . . . . .

Le Bons Massenpsychologie - Le Bon-Rezeption in Deutschland am Beispiel Heinz Marrs - Theodor Geigers Kritik an Le Bon - Neuansatz der Arbeiten Kracauers, Ortega y Gassets und Jaspers' - Kracauers "Massenornament « Ortega y Gassets "Aufstand der Masse « - Jaspers' existenzphilosophische Kritik an der Massengesellschaft - Resümee

b) Literarische Konsequenzen . . . . . . . . . . . . . . . .

Hofmannsthals elitärer Pessimismus bezüglich der Möglichkeiten einer gemeinsamen Sprache - Döblins Forderung nach einer Demokratisierung der Bildung Konsequenz des »bürgerlichen « Massenbegriffs für die politisch-literarische Produktion - Erscheinungsorte politischer Aufsätze: Sammelband und Zeitschrift Stichworte zur Geschichte wichtiger Zeitschriften - "Deutsche Rundschau * "Neue Rundschau " - Charakter der Rundschauzeitschriften - Neuer Zeitschriftentyp der zwanziger Jahre: "Das Tagebuch « und "Die Weltbühne« - Information durch breite Dokumentation der Fakten: Stefan Großmann, Leopold Schwarzschild - Ernst Glaeser: Publizistik als Aufklärung - „Die Tat« als rechtes Korrelat zu "Tagebuch « und »Weltbühne« - Die Problematik von Horst Krügers idealtypischer Abgrenzung von "Essay und Publizistik « - Verbindungen zwischen den Rundschauzeitschriften älteren Typs und den Zeitschriften der Weimarer Jahre Von der "Schaubühne« zur "Weltbühne«: Theaterkritik als Vorstufe politischer Publizistik

\section{HauptTEIL}

I. Thomas Mann . . . . . . . . . . . . . . . .

1. Kunstauffassung

Ausgangspunkt: Asthetik des deutschen Idealismus - Thomas Manns Verhältnis zur "Neuen Sachlichkeit" - Kunst als autonomes Spiel - Das Versagen solcher Kunst in Krisensituationen - Politische Aktivität des Künstlers außerhalb des Kunstbereiches - Problematik des Politik treibenden Künstlers - Präferenz der künstlerischen vor der politisch-essayistischen Produktion: Thomas Manns Schweigen nach der Machtergreifung bis 1936 - Divergierende Urteile über die politische Funktion des epischen und des essayistischen Werkes Thomas Manns

\section{Stellung zum Publikum .}

Die Bedeutung des Erfolges der "Buddenbrooks - Schreiben als ein Leistungsnormen unterworfener »bürgerlicher « Beruf - Kriegsdienst mit der Feder - Rückzug aus gesellschaftlicher Verpflichtung während des Ersten Weltkrieges - Erklärung der "Betrachtungen « zur ausschließlich autobiographischen Künstlerschrift "Romantische« Weltflucht und "öffentliche Einsamkeit « - Repräsentative Rolle erfolgreicher Vorkriegsautoren in der Weimarer Republik - "Der deutsche 
Dichter entdeckt seine Sozialität« - Widerspruch zwischen Thomas Manns gesellschaftlichen Einsichten und seiner schriftstellerischen Praxis - Monolog statt Dialog - Adressat: die bürgerliche Oberschicht - Wiener Rede vor Arbeitern Herablassende Haltung zur Arbeiterschaft - Thomas Manns Attitüde im Spiegel der Kritik Joseph Roths

\section{Darstellung der politischen Essayistik}

"Humanität« als Leitmotiv des Thomas Mannschen Werkes - Die gegenaufklärerische "Humanität« der "Betrachtungen « - Die Antithese "sozial «—»metaphysisch « - Der erweiterte Humanitätsbegriff der zwanziger Jahre - Die Synthese der »deutschen Mitte - "Humanität« und "Republik « - Gruppierung seiner politischen Essays um Rathenaus Ermordung (1922) und die Septemberwahl 1930 - „Von deutscher Republik« (1922) als wichtigster Essay der ersten Gruppe - Novalis-Exegese zur Herstellung eines Sinnbezuges zur Vergangenheit - Hölderlin-Zitat zum Verfassungstag 1924 - "Deutsche Ansprache" (1930) Synopse eines Redeabschnittes mit einem Leitartikel Leopold Schwarzschilds Bündnis zwischen Bürgertum und SPD - Thomas Manns "unpolitischer « Sozialismusbegriff - Die Entwicklung der politischen Essayistik von spolitischer Dichtung zu >literarischer Politik « - Kritik jüngerer Schriftsteller (Joseph Roth, Bertolt Brecht)

\section{Heinrich Mann}

1. Kunstauffassung

Einheit von Kunst und Politik - "social control theory « - Beispiel Balzac "Der Untertan« und der "Zola«-Essay - Heinrich Mann im "Politischen Rat geistiger Arbeiter « - Ablehnung der Russischen Revolution - Fehleinschätzung der Nachkriegssituation in Deutschland - Die "Diktatur der Vernunft« (1923) als Antwort auf das Scheitern aktivistischer Geistpolitik - Dualismus "Geist « und "Staat « - Kritik am "Staat« - Verlagerung der Rolle der Kunst: statt Antizipation gesellschaftlicher Entwicklungen Linderung gegenwärtigen Unbehagens

\section{Stellung zum Publikum}

Heinrich Manns Eintreten für eine demokratische Massenkultur - Volksbegriff (Rousseau, Michelet) - Kritik an Treitschke - "Macht" als Prinzip obrigkeitlicher, "Glück « als Ziel demokratischer Politik - Antithese von "Macht« und "Geist« — "Geist « im Bündnis mit dem "Volk « — "Volk « als überindividuelles Subjekt der Geschichte contra Selbstherrlichkeit sgroßer Männer - Hillers Geistpolitik: Herrschaft anstelle des Volkes - Bei Heinrich Mann Herrschaft des Geistes als Funktion des Volkes - Hillers und Heinrich Manns unterschiedliche Geschichtsphilosophie am Beispiel der Französischen Revolution - Schriftsteller als "Bruder des letzten Reporters - Publizität als literarisches Prinzip - Demokratisierung der Literatur zwecks Erziehung der Masse - Kritik am Bürgertum und Bekenntnis zum Proletariat nach der Machtergreifung

3. Darstellung der politischen Essayistik . . . . . . . . . . . .

Chronologische Übersicht über Heinrich Manns politische Essayistik - Systematische Einteilung der politischen Schriften - Die Schriftsteller-Essays - Die biographischen Essays des Weltkriegsjahrzehnts - Die "Verbandsreden « der zwanziger Jahre - Die Aufsätze der zwanziger Jahre zur französischen Literatur Die tagespolitischen Schriften - Die historisch-politischen Analysen - "Kaiserreich und Republik « - \#Bekenntnis zum Ubernationalen« 
III. Kurt Tucholsky . . . . . . . . . . . . . . . . . . .

1. Kunstauffassung

Skepsis als Grundzug von Tucholskys Kunstauffassung - Im Gegensatz zu Heinrich Mann deutlichere Trennung $z$ wischen politischer Schriftstellerei und Kunstliteratur - "Gebrauchslyrik * - Bevorzugung zeitgebundener Schriftstellerei gegenüber autonomer Dichtung - Skepsis hinsichtlich der Wirkungsmöglichkeiten der »Kunst« - Tucholskys distanzierte Stellung zum Expressionismus und Aktivismus - "Wir Negativen" - Affirmatives Pathos bei Tucholsky

2. Stellung zum Publikum . . . . . . . . . . . . . . . .

Forderung nach Einbezug der Massen in das literarische Leben schon vor dem Ersten Weltkrieg - Skepsis nach Kriegsende - Reflexion der einer Offnung des Publikums entgegenstehenden Gründe - Ausgangspunkt seiner Reflexion der Sprachbarrieren: eine an Schopenhauer geschulte Sprachkritik - Warnung vor "Snobismus der schwieligen Faust « - Kritik der Bildungsprotzerei - Kritik der »Essayisten « - Kein Anspruch Intellektueller auf Führungspositionen innerhalb der Arbeiterbewegung - Schriftsteller nicht Führer, sondern höchstens Helfer des Proletariats

3. Darstellung der politisch-publizistischen Essayistik . . . . . . . .

In der "Weltbühne" vorwiegend kurze Texte - Plan einer Monatszeitschrift für längere Aufsätze - Längere Aufsätze in der "Weltbühne« über Offiziere, Richter und Beamte als Serien - Der Zusammenhang dieser Serien mit Tucholskys politischen Forderungen nach Rathenaus Ermordung - Methode der Untersuchungen: parteiliche Generalisierung - "Militaria « (1919) - "Deutsche Richter « (1927) - »Die Beamtenpest« (1928) - Studie "Die Anstalt« (1929) als Resümee der drei Serien und als Paradigma seiner soziologischen Methode - "Die Anstalt « und "Schloß Gripsholm» - Tucholskys persönliche Perspektive

IV. Ernst Jünger . . . . . . . . . . . . . . . . . . .

\section{Kunstauffassung}

Jüngers vorgeblicher Bruch mit allen »bürgerlichen « Traditionen - "Krieger « und "Arbeiter « als Nachfolger des "Bürgers« - Prüfstein: das Verhältnis zum "Elementaren" - "Individuum* und "Typus - Die "Gestalt« - Marxismus und Sozialismus für Jünger »bürgerliche « Strömungen - Deshalb Emanzipation der Arbeiterschaft aus seiner Sicht sinnlos - Jüngers Ablehnung der "social control theory «: Kunst »nicht Mittel, sondern Objekt der Veränderung" - Ablehnung der »bürgerlichen « Kunst wegen individueller statt typischer Fragestellungen Kritik des Geniekultes - Statt "musealen Betriebs« bürgerlicher Kultur "Kunst als Gestaltung der Arbeitswelt — Parallelen und Gegensätze zur »Neuen Sachlichkeit « - Kunst der Zukunft vor allem Architektur und Landschaftsplanung Funktion der Literatur dagegen statt Gestaltung Orientierung - Zwei Realitätsebenen: Bild des Kreises mit Peripherie und Mittelpunkt - Entsprechend zwei Erkenntniswege: der logisch-diskursive und der intuitive - Für Intuition Bild des "Hauptschlüssels" - Zwei Bereiche der Literatur: sachliche Beschreibung der »Peripherie « (entsprechend dem "neusachlichen « Stilideal) und zum "Mittelpunkt * vorstoßende Dichtung (in Besonderheit die Lyrik) - Kreuzung beider Erkenntniswege: "Stereoskopie* ("magischer Realismus«) - "Stereoskopie« und »doppelter Blick * 
2. Stellung zum Publikum . . . . . . . . . . . . . . . .

Den zwei Erkenntniswegen entsprechend zwei verschieden große Publikumskreise - Elite und Masse - Frontkämpferelite - Ursprünge von Jüngers Elitebegriff Das Kapitel »Untereinander « in "Der Kampf als inneres Erlebnis« in der Erstfassung 1922 - Änderungen späterer Ausgaben - Gründe für die skizzierte Verschiebung des Jüngerschen Elitebegriffs - Vernichtung der »bürgerlichen « Kultur- und Bildungswerte parallel zur Abdankung der sozialen und intellektuellen Elite - „Über den Schmerz« - Jüngers mangelnde Bereitschaft zum geforderten Bildungsverzicht hinsichtlich seiner eigenen Person - Verteidigung seiner Position im »Waldgang « - Die Jünger prägende Erfahrung: die militärische Gliederung in Mannschaft und Führung - Entsprechend zweigleisig sein Verhältnis zum Publikum - Demagogische Publizistik für die Masse - Essayistik für den kleinen Kreis Gebildeter

3. Darstellung der politischen Publizistik und Essayistik . . . . . .

"Der Kampf als inneres Erlebnis« - Dämonologische und naturwissenschaftliche Studien - Nationalrevolutionäre Publizistik — "An die Freunde« - Religiöse Sprache - Methode der Jüngerschen Publizistik - Jüngers Verhältnis zur Technik und zur Großstadt in seinen Aufsätzen - "Programm« der Jüngerschen Publizistik - Kritik an Jüngers Position von rechts und von links - Jüngers "Studien" - Vorwort zu »Krieg und Krieger* - "Feuer und Bewegung * "Die Totale Mobilmachung" - "Der Arbeiter« - Jüngers Essayistik - Vorwort zur zweiten Fassung des "Abenteuerlichen Herzens« - Jüngers Essayistik als Gespräch mit Gleichgesinnten - Verstummen des Publizisten (und »Politikers«) Ernst Jünger

Schlußbemerkungen . . . . . . . . . . . . . . . . . . .

Unwägbarkeit der Wirkung politischer Schriften - Topos der deutschen Diskussion um die Weimarer Republik: Verursachung ihres Unterganges durch linken und rechten Extremismus - Gegenposition: Die Schwäche der demokratischen Mitte als Ursache des Scheiterns der Republik - Hypothese für die Erfolglosigkeit der politisch-literarischen Bemühungen der Brüder Mann und Tucholsky Steuerung der öffentlichen Meinung durch Interessenvertreter (Beispiel Werkspresse) - Erik Regers "Union der festen Hand " (1931) - Regers Bericht über "Die wirkliche Arbeiterpresse « und deren Stellenwert innerhalb der Weimarer Republik

Anmerkungen . . . . . . . . . . . . . . . . . . . . .

(mit Bemerkungen zur Zitierweise und Aufschlüsselung der benutzten Siglen)

Literaturverzeichnis . . . . . . . . . . . . . . . . . .

Personenregister 